\title{
The Molecular Pathology of Odontogenic Tumors: Expanding the Spectrum of MAPK Pathway Driven Tumors
}

\author{
Letícia Martins Guimarães ${ }^{1}$, Bruna Pizziolo Coura ${ }^{1}$, Ricardo Santiago Gomez ${ }^{2}$ and \\ Carolina Cavalieri Gomes ${ }^{1 *}$ \\ 1 Department of Pathology, Biological Sciences Institute, Universidade Federal de Minas Gerais, Belo Horizonte, Brazil, \\ ${ }^{2}$ Department of Oral Surgery and Pathology, Faculty of Dentistry, Universidade Federal de Minas Gerais, Belo Horizonte, \\ Brazil
}

Odontogenic tumors comprise a heterogeneous group of lesions that arise from the odontogenic apparatus and their remnants. Although the etiopathogenesis of most odontogenic tumors remains unclear, there have been some advances, recently, in the understanding of the genetic basis of specific odontogenic tumors. The mitogen-activated protein kinases/extracellular signal-regulated kinases (MAPK/ERK) pathway is intimately involved in the regulation of important cellular functions, and it is commonly deregulated in several human neoplasms. Molecular analysis performed by different techniques, including direct sequencing, next-generation sequencing, and allele-specific qPCR, have uncovered mutations in genes related to the oncogenic MAPK/ERK signaling pathway in odontogenic tumors. Genetic mutations in this pathway genes have been reported in epithelial and mixed odontogenic tumors, in addition to odontogenic carcinomas and sarcomas. Notably, B-Raf proto-oncogene serine/threonine kinase (BRAF) and KRAS proto-oncogene GTPase (KRAS) pathogenic mutations have been reported in a high proportion of ameloblastomas and adenomatoid odontogenic tumors, respectively. In line with the reports about other neoplasms that harbor a malignant counterpart, the frequency of BRAF p.V600E mutation is higher in ameloblastoma (64\% in conventional, $81 \%$ in unicystic, and $63 \%$ in peripheral) than in ameloblastic carcinoma (35\%). The objective of this study was to review MAPK/ERK genetic mutations in benign and malignant odontogenic tumors. Additionally, such genetic alterations were discussed in the context of tumorigenesis, clinical behavior, classification, and future perspectives regarding therapeutic approaches.

Keywords: genetic mutations, BRAF, KRAS, ameloblastoma, benign tumors, ameloblastic, odontogenic, MAPK

\section{INTRODUCTION}

Odontogenic tumors are uncommon lesions that originate from cells and tissues involved in odontogenesis and from their remnants. These tumors comprise a heterogeneous group of lesions ranging from hamartomatous ones to benign and malignant neoplasms [1]. Odontogenic tumors are classified into epithelial, mesenchymal, and mixed epithelial and mesenchymal tumors based on the odontogenic tissue they mimic [1]. 
Reciprocal signaling between epithelium and ectomesenchyme guides the process of tooth embryonic development, which is fully dependent on Wnt, BMP, FGF, Shh, and Eda signals [2]. The pathogenesis of odontogenic tumors is associated with alterations in components of signaling pathways. For instance, studies in the last decade have described pathogenic mutations in mitogen-activated protein kinases/extracellular signal-regulated kinases (MAPK/ERK) pathway cascade components in benign and malignant odontogenic tumors [3].

MAPK/ERK is an intracellular signaling pathway highly dependent on intracellular protein kinases. The activity of the pathway is closely related to the regulation of fundamental cellular functions such as proliferation, survival, growth, metabolism, migration, and differentiation, and alterations in this pathway could contribute to the success of neoplastic cells [4]. Although MAPK/ERK is commonly deregulated in several human cancers, the prognostic and predictive values of each mutation are context-dependent [4]. Notably, mutations in some components of this pathway, such as B-Raf proto-oncogene serine/threonine kinase (BRAF) and KRAS proto-oncogene GTPase (KRAS) genes, are oncogenic drivers but can also be identified in benign and potentially malignant conditions $[3,5,6]$ and even in healthy tissues $[7,8]$.

Although the molecular pathogenesis of odontogenic tumors has not been completely elucidated, it is known that MAPK/ERK signaling pathway plays a role in the molecular pathogenesis of a number of them. Mutations in the MAPK/ERK pathway genes have been reported in ameloblastoma, adenomatoid odontogenic tumor, ameloblastic fibroma, ameloblastic fibrodentinoma, ameloblastic fibro-odontoma, ameloblastic carcinoma, clear cell odontogenic carcinoma, and ameloblastic fibrosarcoma [9-13].

The understanding of the core genetic changes reported in odontogenic tumors could refine their classification, aid in the diagnoses of challenging lesions, and help in the design of new targeted therapies for aggressive and/or malignant cases. Therefore, the present study has aimed to review the alterations in MAPK/ERK components reported for benign and malignant odontogenic tumors. Furthermore, we review MAPK signaling, discuss the described MAPK/ERK genetic alterations in the context of tumorigenesis, biological behavior, entity classification, and future perspectives regarding targeted therapy.

The search strategy included the terms "odontogenic tumor," "odontogenic tumour," "MAPK," "MAPK/ERK," "mutation," "FGFR,” "BRAF," “KRAS," "NRAS," “HRAS," “ameloblastoma," "adenomatoid odontogenic tumor," "ameloblastic fibroma," "ameloblastic fibrodentinoma," "ameloblastic fibro-odontoma," "ameloblastic carcinoma," "clear cell odontogenic carcinoma," and "ameloblastic fibrosarcoma" which were connected with appropriate Boolean operators "AND" and "OR". Important papers from the background knowledge and the reference list of the studies were also added. All studies that used molecular techniques and/or immunohistochemistry to assess MAPK/ERK mutations in odontogenic tumors were included.

\section{MAPK SIGNALING PATHWAYS}

The MAPK signaling pathways are master regulators of multiple cellular responses whose activation is triggered by a wide variety of extracellular signals [4]. Transmembrane receptors can recognize stimuli, including hormones, growth factors, mitogens, and inflammatory cytokines, and transmit signals from the extracellular membrane to the nucleus through phosphorylation cascades which then regulate fundamental cellular functions [4].

Eukaryotic cells have at least seven MAPK pathways named according to their final regulatory proteins [14]. They are classified as conventional or atypical mainly based on the activation mechanisms [14]. Each MAPK pathway has a specific mode of activation and downstream functions due to variations in protein expression, subcellular compartmentalization, interaction in protein complexes (scaffolds proteins) partners, and substrate-targeting mechanisms leading to distinct cellular responses [4]. Among the conventional MAPK pathways, extracellular regulated kinases (ERK1/2), Jun $\mathrm{NH} 2$ terminal kinases (JNK1/2/3), and p38 (p38 $\alpha / \beta / \gamma / \delta)$ are the most well-characterized groups $[4,15]$.

Even though each MAPK pathway is unique, they share the presence of three evolutionarily conserved effectors, which are sequentially acting protein kinases generically known as MAPKKK, MAPKK, and MAPK [15-17]. In the canonical MAPK/ERK signaling pathway (Figure 1A), specifically in the RAS-RAF-MEK-ERK cascade, it is notable that these intracellular effectors correspond to RAF (MAPKKK), MEK1/2 (MAPKK), and ERK1/2 (MAPK) proteins. They are further upregulated by the RAS GTPases family (RAS). RAS proteins are located at the inner surface of the cell membrane and maintain balance through cycling between their inactive and active states through binding to guanosine diphosphate (GDP) and guanosine triphosphate (GTP), respectively. The conversion from its stable and quiescent cytoplasmic form to the active form is catalyzed by guanine nucleotide exchange factors (GEFs) through reversible phosphorylation reaction, therefore leading to downstream pathway activity. GTP-loaded RAS recruits and interacts directly with RAF protein, promoting its activation through complex processes that culminate in dimerization of the RAS-RAF binding domain. Activated RAF phosphorylates MEK within the activation segment and promotes the recruitment and activation of ERK through phosphorylation on both threonine and tyrosine sites. Once activated, ERK phosphorylates RSK, MSK, and MNK cytoplasmic targets, and translocates to the nucleus where it finally has access to nuclear substrates. Transcription factors, other proteins which include kinases and phosphatases, and cytoskeletal elements are some nuclear targets involved in MAPK/ERK cascade. The ERK phosphorylation-induced activity usually results in the activation of these substrates. However, it can degrade and inhibit them in some cases. Usually, after suspension of the external stimulus, the active form of RAS switches to the inactive state of RAS by hydrolysis of GTP due to the direct interaction of GTPase-activating proteins (GAPs) with RAS-GAP complex formation. This complex formation, followed by dephosphorylation reactions mediated by MAPK 


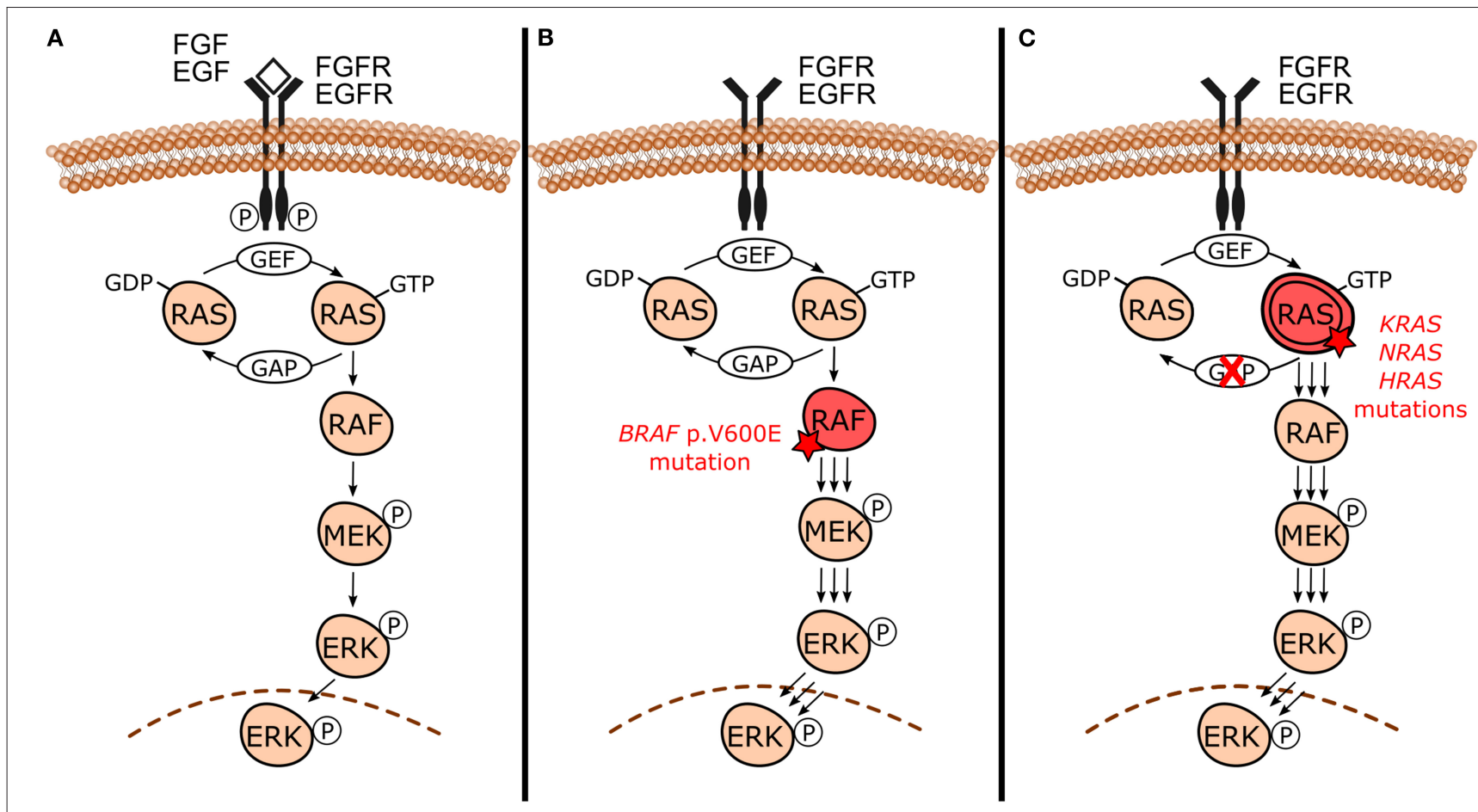

FIGURE 1 | Canonical mitogen-activated protein kinases/extracellular signal-regulated kinases (MAPK/ERK) signaling pathway under normal circumstances and in the presence of activating mutations. (A) Canonical RAS-RAF-MEK-ERK cascade and its normal activation by external signals (e.g., FGF and EGF growth factors) binding to receptor tyrosine kinases [e.g., fibroblast growth factor receptor (FGFR) and epidermal growth factor receptor (EGFR)]. This interaction triggers signaling through RAS-GTP, RAF, MEK, and ERK culminating with the action of phosphorylated ERK in its substrates and the regulation of cellular biological functions. In the absence of external stimulus, active RAS-GTP switches to its inactive form RAS-GDP by hydrolysis due to GAPs action. (B) In the presence of BRAF p.V600E mutation, BRAF constitutively activates MAPK/ERK signaling even in the absence of growth factors and dimerization with RAS, sustaining MAPK/ERK signaling. (C) Activating mutations in RAS genes (KRAS, NRAS, and HRAS) lead to unbalance between inactive and active RAS forms toward the active state (RAS-GTP) either by reducing GTP hydrolysis or by increasing the rate of GTP loading. This mechanism constitutively activates MAPK/ERK signaling even without external stimulus,

sustaining its signals.

phosphatases and negative feedback, are crucial components involved in the inactivation of the pathway $[4,15,18,19]$.

MAPK/ERK pathway coordinates six essential physiological processes in response to extracellular signals, including cell proliferation, survival, growth, metabolism, migration, and differentiation $[4,15,18,19]$.

Generally speaking, MAPK signaling cascades appear to have simple and linear biochemical actions, with the unidirectional activity of protein kinases. Nevertheless, the pathway is highly intricate and the complexity and pleiotropism of the MAPK/ERK pathway are genuinely linked to the multiple subsets of ERK substrates. Diversity of MAPK components isoforms, duration, and intensity of signals, protein localization in different cell compartments, scaffold proteins, and cross-talk are the mechanisms that enhance the range of the regulatory activity of the MAPK/ERK pathway $[4,15,18,19]$.

Alterations in MAPK/ERK components in the different tiers of the cascade culminate in the constitutive activation of the pathway. This leads to abnormal crucial cellular functions that can significantly contribute to the evolutionary advantages of neoplastic cells when compared to surrounding cells [20, 21]. Indeed, genetic studies have shown that MAPK/ERK is commonly deregulated in many human diseases, including human cancers and RASophaties $[4,15]$.

Although extensive molecular analyses have focused on cancer molecular investigations, recent advances in genome screening and other gold-standard molecular techniques have aided to elucidate the molecular basis in a non-malignant context. Benign and potentially malignant lesions can harbor oncogenic mutations once considered exclusive of cancer. Furthermore, these mutations sometimes occur at a higher frequency in the benign lesions than in their corresponding malignant counterparts $[3,5,6]$.

Odontogenic tumors have been shown to harbor high frequencies of both BRAF and RAS mutations [9-13]. The $B R A F$ gene encodes the BRAF protein, which is a RASregulated cytoplasmic serine-threonine kinase that activates the MAPK/ERK signaling pathway by phosphorylating MEK [4, 22]. The gene $B R A F$ is the only member of the $R A F$ gene family to be frequently activated by mutations in human neoplasms [23]. The missense BRAF mutation that results in a valine $(\mathrm{V})$ to glutamic acid (E) substitution at codon 600 (BRAF p.V600E) constitutively activates MAPK/ERK pathway [21] (Figure 1B). Aside from stimulating MAPK/ERK signaling, BRAF p.V600E induces cell 
proliferation and is capable of promoting transformation, which collectively supports its classification as an oncogene [24]. In an experimental model using Braf p.V600E knock-in mouse, expression of the mutation in all tissues lead to embryonic lethality [25]. Therefore, it is unlikely that BRAF p.V600E mutation is compatible with life in humans. Since 2002, BRAF pathogenic mutations frequencies and their implications have been studied in a wide variety of tumor types wherein the majority specifically involve the BRAF p.V600E mutation [26, 27]. BRAF gene mutation has been identified in about $6 \%$ of human cancers [27], including melanomas (40-50\%) [2729], thyroid cancers $(10-70 \%)$ [27, 30], colorectal cancers $(\sim 10 \%)[27,31,32]$, and non-small cell lung cancer $(3-5 \%)$ $[27,33]$.

KRAS, NRAS proto-oncogene GTPase (NRAS), and HRas proto-oncogene GTPase (HRAS) are members of RAS protooncogenes family $(R A S)$ and they play an important role in human cancers [34]. These genes encode RAS GTPases, which are upstream activators of MAPK/ERK and act by interacting and activating RAF proteins. RAS mutations occur in $30 \%$ of human malignant neoplasms and KRAS is the most frequent mutated oncogene. KRAS codon 12 mutations correspond to nearly $90 \%$ of all KRAS mutations in pancreatic, colorectal, and lung human cancers [34]. RAS mutations constitutively activate MAPK/ERK pathway, as shown in Figure 1C.

Interestingly, some studies have also reported somatic oncogenic mutations in normal endometrium and breast cells of healthy women $[7,8]$. The study of Coura and colleagues (2020) investigated the presence of KRAS p.G12V and p.G12R and BRAF p.V600E mutations in normal odontogenic tissue remnants, specifically in dental follicles associated with unerupted teeth, as an attempt to uncover early oncogenic mutations that could lead to odontogenic tumors tumorigenesis. However, these specific KRAS and BRAF mutations have not been detected in any of the investigated samples [35]. Therefore, despite the presence of MAPK/ERK oncogenic mutations in odontogenic tumors, the role of such mutations play in the pathogenesis of these lesions remains to be clarified.

\section{MAPK/ERK Signaling Pathway Cross-Talk}

Cross-talk between MAPK cascades and other cellular regulatory pathways can extensively influence MAPK/ERK signaling by dynamic and complex interactions $[4,36]$.

Illustrating such phenomena, cross-talk between MAPK/ERK and PI3K/AKT/mTOR pathways at different stages of signal propagation can amplify key target regulatory protein activities through cross-activation and/or substrate convergence $[4,36]$. MAPK/ERK regulates PI3K, TSC, and mTOR activity by activating PI3K and $\mathrm{mTORC} 1$ and inhibiting TSC2 $[4,36]$. In this case, an important positive loop involves PI3K activation and GAB docking proteins phosphorylation. Once phosphorylated, GAB interferes in RAS-GAP complex binding, decreasing RAS inactivation and leading to positive ERK upstream regulation [36]. Moreover, ERK, RSK, AKT, and S6K protein kinases, and the components of MAPK/ERK and PI3K/AKT/mTOR pathways, often phosphorylate the same substrate including FOXO and c-Myc transcription factors $[4,36]$.

It is also known that the $\mathrm{WNT} / \beta$-catenin signaling pathway interferes in MAPK/ERK regulation. The active GSK3 $\beta$ in the destruction complex (binding to $\beta$-catenin, APC, and AXIN) phosphorylates RAS, which is consequently degraded. Aberrant activation of the $\mathrm{WNT} / \beta$-catenin pathway leads to excessive dissociation of destruction complex with consequent accumulation of $\beta$-catenin and RAS proteins in the cytoplasm. This promotes the $\beta$-catenin translocation to the nucleus and facilitates MAPK/ERK downstream activity by increasing RAS availability [37, 38]. In that case, mutual deregulation in MAPK/ERK and hyperactivation of WNT/B-catenin seems to cooperatively act triggering tumorigenesis $[37,38]$.

\section{MAPK/ERK Pathway in Odontogenesis}

Interactions between epithelial and ectomesenchymal cells govern tooth development during odontogenesis. The molecular mechanisms associated with odontogenesis involve intracellular signaling cascades, including MAPK, Hedgehog, and Wnt pathways, and alterations in these pathways have been associated with the pathogenesis of odontogenic lesions [2, 3]. It is beyond the scope of this review to discuss the Hedgehog and Wnt pathways and the molecular pathogenesis of odontogenic lesions related to them. Thereby, we focused on the prototypical MAPK/ERK signaling pathway and the odontogenic tumors associated with its disturbance.

MAPK/ERK signaling plays a role in odontogenesis [39]. Unlike humans, mice are monophyodonts and have intrinsic differences from humans. However, mice dentition is a useful model to study the mechanisms related to human dental development [39, 40]. Supporting a role for MAPK/ERK signaling in odontogenesis, mice carrying deletions in Sprouty genes, which encode negative regulators of MAPK/ERK pathway, have hyperactive MAPK/ERK pathway signaling and supernumerary teeth $[39,41]$. Similarly, ribosomal protein S6 kinase (RSK) mutant mice develop supernumerary teeth and alteration in dental shape patterns $[39,40]$. RSKs are protein kinases that act downstream of the MAPK/ERK cascade and seem to have a feedback inhibitory effect on MAPK/ERK signaling $[39,40]$. It is notable that the mutations in RSK2 cause Coffin-Lowry syndrome (OMIM \#303600), in which dental anomalies can be present [40].

In addition, experimental data support the association of MAPK/ERK with odontogenic tumors tumorigenesis through $R A S$ genes. Notably, Hras-G12V mutant mice show defects in the differentiation and proliferation of ameloblasts and their precursors [42, 43], while Hras transgenic mice develop jaw tumors consistent with odontogenic tumors [44-46]. Further supporting a role for MAPK/ERK signaling in odontogenesis, KRAS, RAF1, MEK1, and ERK1/2 protein expressions have been detected in human tooth germs by immunohistochemistry [47].

The fibroblast growth factor receptor 1 (FGFR1), which is a receptor whose stimulation activates MAPK/ERK pathway, has a strong expression in mouse odontoblasts, in addition to FGFR2 (isoform IIIb) in ameloblasts. This suggests that the fibroblast growth factors (FGFs) have a role in the regulation 


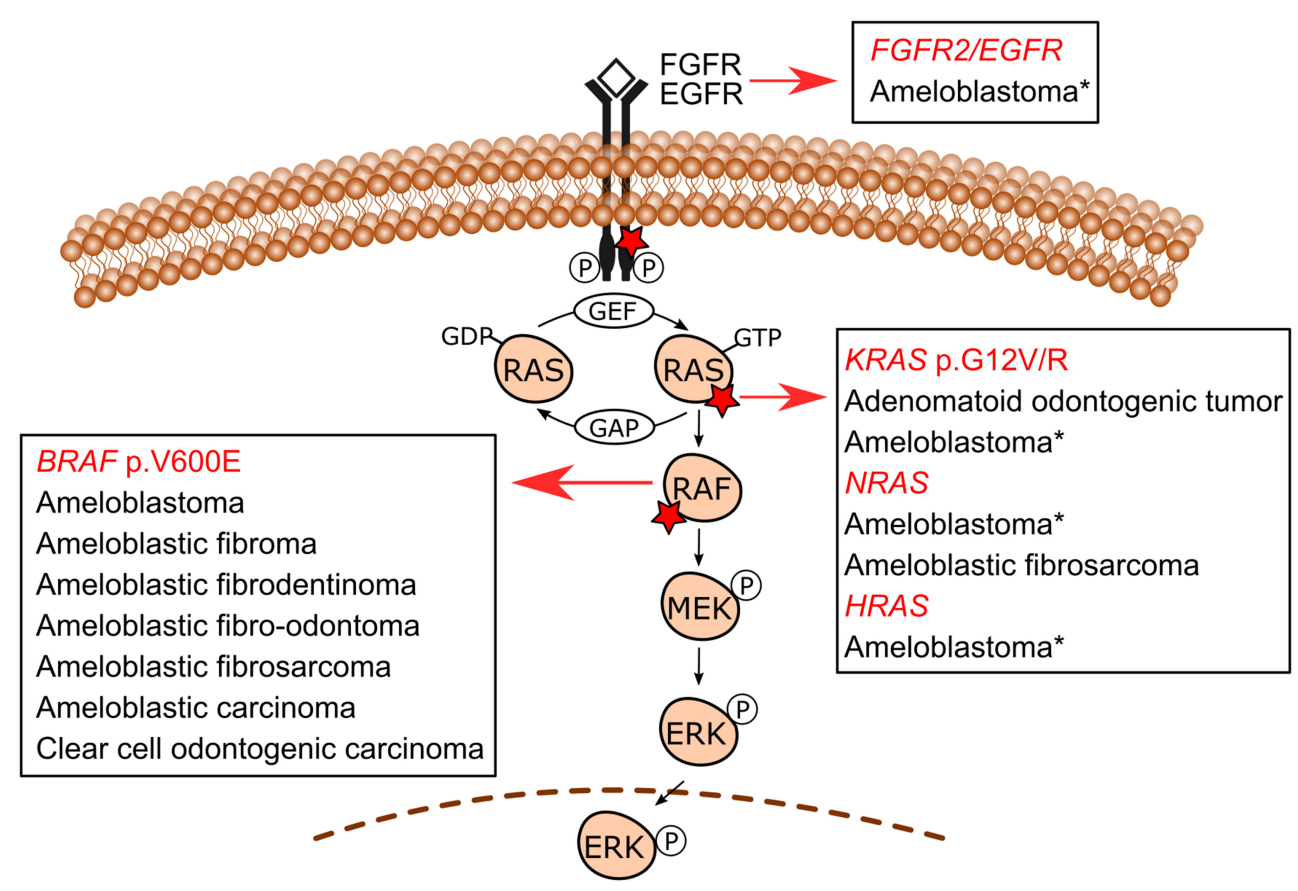

FIGURE 2 | MAPK/ERK signaling pathway mutations in odontogenic tumors. BRAF p.V600E is the most common mutation in ameloblastomas, followed by KRAS (mostly p.G12R), NRAS, HRAS, and FGFR2 mutations reported in a few BRAF wild-type cases. Additionally, an EGFR mutation has also been reported in one ameloblastoma case. These less commonly reported mutations in ameloblastomas are indicated with an asterisk (*). Ameloblastic fibromas, ameloblastic fibrodentinomas, ameloblastic fibro-odontomas, ameloblastic fibrosarcoma, ameloblastic carcinomas, and clear cell odontogenic carcinoma (a single case) also carry BRAF p.V600E mutation. An NRAS mutation has also been reported in one case of ameloblastic fibrosarcoma in a mutually exclusive manner with BRAF p.V600E. Adenomatoid odontogenic tumors are characterized by frequent KRAS codon 12 (either p.G12V or p.G12R, and in a single case p.G12D) driver mutations, which occur in approximately $70 \%$ of cases. Detailed information on the studies reporting these mutations is shown in Table $\mathbf{1 .}$

of their differentiation and secretory functions [48]. Moreover, the expression patterns of different FGFs in dental epithelium and mesenchyme are dynamic, supporting the existence of regulatory signaling cascades between FGFs in both these tissues during odontogenesis [49]. Another receptor whose stimulation activates MAPK/ERK pathway is the epidermal growth factor receptor (EGFR). There is consistent evidence that EGFR expression during odontogenesis has an important role during this developmental process [50].

Collectively, the above-mentioned studies support a role for MAPK/ERK signaling pathway in odontogenesis regulation, and disturbance in this pathway may lead to either odontogenesis impairment or can give rise to odontogenic tumors in animal models.

\section{MAPK/ERK MUTATIONS IN ODONTOGENIC TUMORS}

The use of molecular pathology techniques has led to the identification of MAPK/ERK-related gene mutations in specific odontogenic tumors. Initially, such mutations have been uncovered in benign epithelial odontogenic tumors wherein $B R A F$ and KRAS mutations were revealed in ameloblastomas and adenomatoid odontogenic tumors, respectively $[9,11]$. In recent studies, mixed odontogenic tumors and malignant odontogenic tumors have also been included in the spectrum of MAPK pathway-driven tumors. Figure 2 shows the MAPK/ERK genes for which mutations have been described in odontogenic tumors. Table 1 summarizes the frequency of mutations in each tumor.

The following sections addressed the main genetic alterations reported for ameloblastomas, mixed odontogenic tumors, odontogenic carcinomas, where BRAF p.V600E mutation is the most important one, and adenomatoid odontogenic tumors which are characterized by KRAS mutations.

\section{Ameloblastoma}

Ameloblastoma is a locally aggressive benign epithelial odontogenic tumor occurring mainly in the posterior mandible. Although benign, this tumor can behave aggressively and recur. Surgical approaches to remove this tumor often lead to facial deformity and morbidity [1]. Ameloblastomas are differently categorized into ameloblastoma (conventional), unicystic type, extraosseous/peripheral type, and metastasizing ameloblastoma, with distinct biological behaviors [1].

In 2014, the pioneer study by the group of Heikinheimo reported for the first time recurrent activating BRAF p.V600E mutations in ameloblastomas [9]. Considering that MAPK/ERK signaling can be activated by stimulation of transmembrane receptors, including EGFR, and that overexpression of EGFR had previously been reported in ameloblastomas, when 
TABLE 1 | Summary of MAPK/ERK mutations and their frequencies in odontogenic tumors.

\begin{tabular}{lll}
\hline Odontogenic tumors & $\begin{array}{l}\text { Mutations in MAPK/ERK } \\
\text { pathway genes }\end{array} \quad$ Frequency of each mutation ${ }^{\#}$
\end{tabular}

\section{Benign epithelial} odontogenic tumors

Conventional Ameloblastoma

BRAF p.V600E $\quad 64 \%(478 / 746)^{\mathrm{a}}$

[9], [10], [51], [52], [53] , [54],

[55], [56], [57], [58], [59], [60],

[61], [62], [63], [64], [65], [66] $]^{\dagger}$,

$[67]^{\dagger},[68]^{\dagger},[69]^{\dagger},[70]^{\dagger},[71]^{\dagger}$

$\begin{array}{lll}\text { KRAS }^{*} \mathrm{~b} & 7.2 \%(13 / 180) & {[51],[52],[55]^{\mathrm{g}},[62],[72]} \\ \text { NRAS }^{* \mathrm{c}} & 5.2 \%(8 / 155) & {[52],[54],[55]^{\mathrm{g}},[58]} \\ \text { HRAS }^{* \mathrm{c}} & 4 \%(6 / 151) & {[52],[55]^{\mathrm{g}},[58]} \\ \text { FGFR2 }^{* d} & 11.3 \%(14 / 124) & {[51],[52],[58],[72]} \\ \text { EGFR }^{*} & 1.6 \%(1 / 62) & {[55]^{g}}\end{array}$

Unicystic ameloblastoma

BRAF p.V600E

$80.7 \%(109 / 135)$

Peripheral ameloblastoma

BRAF p.V600E

$62.5 \%(10 / 16)$

[10], [55], [58] ${ }^{\mathrm{h}},[60]$, [62], [63],

Adenomatoid odontogenic

NRAS p.Q61R *

$33.3 \%(1 / 3)$

KRAS p.G12V

$42.9 \%(24 / 56)$

[64], [68] $]^{f},[69]^{f},[70]^{f},[71]^{f}[73]$

tumor

KRAS p.G12R

$34 \%(16 / 47)$

KRAS p.G12D

$11.1 \%(1 / 9)$

[54], [55], [62], [69] $]^{f},[71]^{\dagger}$

[54]

[11], [12], [74]

Benign mixed epithelial \&

mesenchymal odontogenic

tumors

Ameloblastic fibroma

BRAF p.V600E

$45.8 \%(11 / 24)$

BRAF p.V600E $\quad 60 \%(3 / 5)$

Ameloblastic fibrodentinoma

BRAF p.V600E

$34.6 \%(9 / 26)$

[12], [74]

[74]

Ameloblastic fibro-odontoma

Odontogenic sarcomas

Ameloblastic fibrosarcoma

$\begin{array}{ll}\text { BRAF p.V600E } & 70 \%(7 / 10) \\ \text { NRAS p.Q61K* } & 14.3 \%(1 / 7) \\ \text { BRAF p.V600E } & \\ \text { BRAF p.V600E } & 35.3 \%(6 / 17) \\ & 100 \%(5 / 5)^{\star *} \\ \end{array}$

[13], [52], [53], [63]

[13], [52]

[13], [53], [63]

Odontogenic carcinomas

Ameloblastic carcinoma

Clear cell odontogenic

carcinoma

aIndividual frequency per study ranged from 30 to 90\%, except for two studies that have reported mutations in all samples probably due to limited sample size [2/2 [64] and 4/4 [65]]. ${ }^{b}$ In eight out of $13(61.5 \%)$ cases the mutation was KRAS p.G12R and in one sample KRAS p.L56_G60dup. For the other cases, the specific mutation was not reported.

${ }^{c}$ With regard to NRAS, p.Q61R was the most frequent mutation, occurring in approximately 50\% of the eight NRAS mutation-positive samples, and NRAS p.Q61K was also reported. With regard to HRAS mutations, HRAS p.Q61R was the most frequent one, occurring in 3/6 (50\%) of HRAS mutation-positive samples, HRAS p.G12S and p.Q61K were reported in one case each, and the other one was not specified.

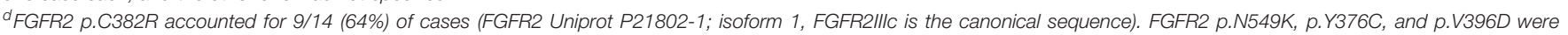
detected in one case each. FGFR2 p.V395D was also reported. Based on the published information we could not ascertain which specific mutation corresponds to the 14th case. Gültekin et al. [55] reported one FGFR mutation in 1/62 cases, however, it is not clear if it occurred in FGFR2. Therefore, we opted to not include this data for FGFR2.

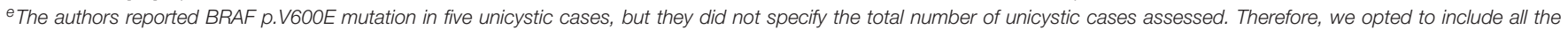
mutation-positive samples (14/19) in the conventional ameloblastoma count.

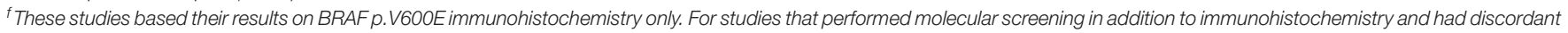
results, we considered the molecular results.

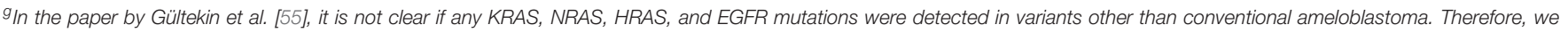
considered the total number of assessed samples $(n=62)$, to calculate the frequency of KRAS, NRAS, HRAS, and EGFR mutations.

${ }^{h}$ Considering only the mandibular location, The study of Heikinheimo et al. [58] described BRAF p.V600E in 29/31 (93.5\%) of unicystic ameloblastomas.

*Mutually exclusive with BRAF p.V600E, except for one case harboring FGFR2 p.C382R and BRAF p.V600E.

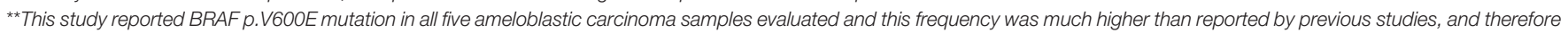
we did not add the results to the other ones when calculating mutation frequency.

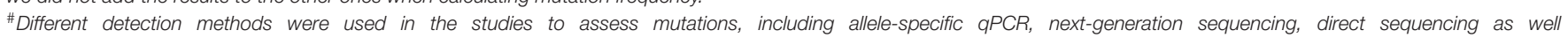
as immunohistochemistry. 
the investigators detected ameloblastoma cultured cells resistant to EGFR-targeted inhibition they screened these cells for BRAF p.V600E mutation [9]. After detecting the mutation in this index case, the study of Kurppa et al. (2014) detected BRAF p.V600E mutation in 62.5\% (15/24) of ameloblastoma samples through Sanger sequencing [9]. In addition, the study of these authors demonstrated the utility of the BRAF p.V600E-specific monoclonal antibody VE1 to detect the BRAF p.V600E mutation in ameloblastomas by immunohistochemistry [9].

The study of Sweeney et al. carried out targeted nextgeneration sequencing (NGS) and/or Sanger sequencing and VE1 immunohistochemistry where they detected BRAF p.V600E mutation in $43 \%(12 / 28)$ of ameloblastoma samples [51]. Additionally, they detected another BRAF variant (p.L597R) in one sample [51]. The study of Brown et al. performed a combination of allele-specific PCR, NGS gene panel, and Sanger sequencing and showed BRAF p.V600E in 62\% (31/50) of ameloblastoma cases [52]. They further reported the presence of BRAF p.V600E mutation in 67\% (23/34) of ameloblastomas by using VE1 immunohistochemistry in cases not suitable for molecular evaluation [52]. Following these pieces of research, several studies have also assessed the presence of $B R A F$ p.V600E in conventional ameloblastoma, either by molecular techniques or a combination of mutation screening and immunohistochemistry, with mutation frequencies varying from 55 to $\sim 90 \%[10,53-63]$. In two studies with limited sample numbers, the mutation was detected in all samples [2/2 [64] and 4/4 [65]]. The frequency of BRAF p.V600E mutation in studies that have assessed the mutation only using immunohistochemistry varied from 33 to $79 \%$ [66-71]. Notably, although immunohistochemical expression of BRAF p.V600E has been used as a surrogate of BRAF p.V600E mutation, results must be interpreted with caution since there are false-positive cases detected by immunohistochemistry and not confirmed by molecular technique [73] as well as false-negative cases [63].

There is no consensus on the association of BRAF p.V600E mutation with ameloblastoma clinicopathological features, including location, age, histology, as well as clinical behavior. In the studies conducted by Sweeney et al. and Gültekin et al., they suggested the predominance of BRAF p.V600E ameloblastomas in mandible when compared to $B R A F$ wild-type tumors $[51,55]$. The studies of Brown et al., Bonacina et al., and Kelppe et al. have also suggested BRAF p.V600E positive cases mostly locate in the mandible and occur at earlier ages [52, 62, 69]. In agreement with that, the study of Gültekin et al. has also reported that BRAF p.V600E ameloblastoma cases occur at a much younger age than BRAF p.V600E wild-type ones [55]. Additionally, $B R A F$ mutations have been reported by some authors to predominantly occur in non-plexiform histologic type ameloblastoma [51, 55, 71], while conversely others reported $B R A F$ mutations predominance in plexiform type [67]. It is worth mentioning that in this last study [67], the authors have assessed the mutation using immunohistochemistry as a surrogate marker for the mutation. However, they used a different antibody clone other than VE1. Nonetheless, most studies reported recurrent $B R A F$ p.V600E mutation regardless of histological type $[9,10,56,60,66]$, mandibular/maxillary location $[10,56,66]$, and the age of patients [9].

The association between the presence of BRAF p.V600E and ameloblastoma aggressiveness is unclear. While some studies had reported earlier recurrence and increased recurrence rates in $B R A F$ wild-type tumors $[52,55,58]$, others had observed a more aggressive behavior with poor disease-free survival and higher recurrence rate in ameloblastomas harboring $B R A F$ p.V600E mutation; in these cases, assessed by immunohistochemistry only $[66,67]$. In recent times, no difference has been found in relapsefree intervals between $B R A F$ p.V600E positive and wild-type ameloblastoma cases [62].

Mutations in 28 genes have been assessed by an NGS targeted panel, in addition to smoothened frizzled class receptor (SMO) mutations, assessed by Sanger sequencing [55]. Mutations have been detected in approximately $90 \%$ of the ameloblastomas, comprised of conventional, unicystic, and peripheral cases, with $B R A F$ p. V600E corresponding to $60 \%$ of mutation-positive cases [55]. SMO mutations have been detected in $14 \%$ of cases, in a mutually exclusive pattern with $B R A F$ mutations. Additionally, NRAS, HRAS, and EGFR somatic mutations have been detected in one case each, mutually exclusive with $B R A F$ and $S M O$ mutations. In a few cases, BRAF or SMO mutations co-occurred with mutations in other genes. Importantly, the authors reported high recurrence rate in ameloblastomas harboring multiple gene mutations (higher mutational burden) detected either by targeted NGS or Sanger sequencing. At the same time, a low risk for relapse was observed in tumors with a single $B R A F$ mutation [55]. It is notable that the presence of two or three gene mutations, including somatic mutations in KRAS, PIK3CA, PTEN, FGFR, CDKN2A, and CTNNB1 in the background of either $B R A F$ or $S M O$ mutation-positive ameloblastomas, exclusively occurred in solid/conventional tumors [55]. Further studies may help to clarify if these associations between tumor mutation burden and aggressiveness hold.

The study of Diniz and co-workers in 2015 tested whether the BRAF p.V600E mutation was a signature of conventional ameloblastoma or if unicystic lesions also harbored this mutation. BRAF p.V600E was detected in $83 \%(5 / 6)$ of unicystic ameloblastomas [10]. In the later study of Heikinheimo et al., they reported BRAF p.V600E mutation in 94\% (29/31) of mandibular unicystic ameloblastomas and higher homogeneity of mutations than that of conventional ameloblastomas, since rare samples carried a mutation other than BRAF [58]. The mutational screening of $B R A F$ by molecular assays has been shown to be useful for the differential diagnosis between unicystic ameloblastomas and odontogenic cysts [64, 73]. Furthermore, a consistent homogeneous profile for BRAF mutational status has been shown in unicystic ameloblastomas, with BRAF p.V600E detection by allele-specific qPCR in all areas with different histological appearances from the same neoplasm, including those areas resembling other odontogenic lesions [64]. Other studies that reported BRAF p.V600E mutation in the unicystic variant by molecular screening and/or immunohistochemistry are listed in Table $\mathbf{1 .}$

A small number of studies have assessed genetic mutations in peripheral ameloblastomas $[54,55,62,69,71]$. The BRAF 
p.V600E was also the most common mutation among them, and it was detected in $62.5 \%(10 / 16)$ of cases $[54,55,62$, 69, 71]. Additionally, single somatic mutations in NRAS [54] and SMO [55] have also been detected in one peripheral ameloblastoma sample each in a mutually exclusive manner with the BRAF mutation.

Mutually exclusive and less common mutations affecting other MAPK-related genes, such as KRAS, NRAS, HRAS, and FGFR2 (a receptor whose stimulation activates MAPK/ERK pathway) have been reported in $B R A F$ wild-type ameloblastomas $[51,52,54,55,58,62,72]$. It is notable that the KRAS p.G12R mutation, which occurs in adenomatoid odontogenic tumors [12], has been reported in 4/50 (8\%) [52] and 4/28 (14\%) [51] of $B R A F$ wild-type ameloblastoma cases. Furthermore, in addition to the above-mentioned mutations in MAPK pathway genes, somatic EGFR mutation has been detected in one single case of maxillary ameloblastoma [55]. Mutations in FGFR2 have been described in 4/28 (14\%) [51], 3/50 (6\%) [53], and 2/39 (5\%) [58] of BRAF wild-type conventional ameloblastomas. The study of Bartels et al. assessed BRAF wild-type ameloblastomas by targeted NGS and identified FGFR2 mutations in 4/7 (57\%) of them, including one FGFR2/TP53/PTEN-triple-mutant case [72]. Additionally, increased proliferation through MAPK/ERK activation of ameloblastomas cultured cells has been shown after treatment with FGF ligands [77], reinforcing the role of FGF signaling in the tumorigenesis of ameloblastomas.

Other cancer-associated mutations not directly involved in the MAPK pathway have also been detected in ameloblastomas [51, 52, 54, 55, 57, 58, 72, 78]. Among them, SMO mutations have been detected in between 13 and $39 \%$ of ameloblastomas, occurring in a mutually exclusive pattern with BRAF p.V600E $[51,52,55]$. SMO mutations often occur along with an additional RAS family or FGFR2 mutation [51, 52, 55]. Interestingly, the study of Sweeney et al. proposed site-specific BRAF and $S M O$ mutations in mandible and maxilla, respectively [51]. This finding was later supported by the study of Gültekin et al. in a larger cohort [55]. However, the site-specificity of these mutations has not been confirmed by other groups [10,58]. Aside from the SMO mutations, APC mutations have been detected in 3/6 ameloblastomas [78], and CTNNB1, PIK3CA, SMARCB1, $P T E N, C D K N 2 A$ mutations have been reported in a few cases and tend to occur in a non-mutually exclusive manner with $B R A F$ p.V600E mutation and with each other [52, 54, 55]. Protein products of some of these genes, e.g., APC, $\beta$-catenin encoded by CTNNB1, and PI3K encoded by PI3KCA, are components of pathways that cross-talk with MAPK/ERK, as discussed in the "MAPK/ERK signaling pathway cross-talk" topic.

Recently, two studies performed whole-exome sequencing to assess coding mutations in ameloblastomas and reported uncommon mutations in several other genes occurring in the background of BRAF p.V600E mutation [57, 65]. The study of Guan et al. reported BRAF p.V600E mutation in $82 \%$ $(9 / 11)$ of samples and proposed intratumor heterogeneity in ameloblastomas based on different variant allele frequencies (VAF) of BRAF p.V600E and other detected mutations [57]. Mutations in the CDC73 gene, which encodes parafibromin, have been detected in 2/11 samples [57]. It is worth mentioning that $C D C 73$ germline mutation causes hyperparathyroidism-jaw tumor syndrome (OMIM \#145001), in which ossifying fibromas may occur. The study of Shi et al. suggested a "two-hits" mechanism contributing to ameloblastoma tumorigenesis, with BRAF p.V600E (detected in all four assessed samples) corresponding to the first hit and mutations in genes belonging to the gene network of cell proliferation, such as HSAP4, corresponding to the second hit [65]. However, further investigations in larger cohorts and using other methodologies for evaluating tumor clonality and the role of the detected mutations in ameloblastoma development, if any, are needed to confirm these findings. Currently, the evidence is still insufficient to draw any conclusions about further mutations other than BRAF p.V600E participating as drivers of ameloblastoma pathogenesis.

\section{Benign Mixed Epithelial \& Mesenchymal Odontogenic Tumors}

The BRAF p.V600E mutations have been reported in benign mixed odontogenic tumors [13, 52, 53, 63], namely ameloblastic fibroma [13, 52, 53, 63], ameloblastic fibrodentinoma [13, 52], and ameloblastic fibro-odontoma [13, 53, 63].

Collectively, these studies reported BRAF p.V600E mutations in $45.8 \%(11 / 24)$ of ameloblastic fibromas [13, 52, 53, 63], $60 \%(3 / 5)$ of ameloblastic fibrodentinomas [13, 52], and $34.6 \%$ $(9 / 26)$ of ameloblastic fibro-odontomas [13, 53, 63], whereas all nine odontoma samples investigated were wild-type for BRAF p.V600E $[13,63]$. These results suggested that at least a subset of ameloblastic fibromas, ameloblastic fibrodentinomas, and ameloblastic fibro-odontomas are pathologic entities distinct from odontomas. However, this subject is still debatable.

It was suggested that the BRAF p.V600E mutation was limited to the epithelial component of the four mutant cases of mixed tumors which had the two components tested separately, i.e., $1 / 1$ mutant ameloblastic fibroma and 3/3 mutant ameloblastic fibro-odontomas [53]. However, our group has recently dealt with this issue using a straightforward methodology. We have subjected mixed odontogenic tumor samples to laser capture microdissection before molecular testing in an attempt to avoid epithelial-mesenchymal cross-contamination and using allele-specific quantitative PCR, a high sensitivity mutation detection assay, we detected the BRAF p.V600E mutation in the mesenchymal component of all mutation-positive mixed odontogenic tumors (9/9) [13]. Notwithstanding, BRAF p.V600E positive status was additionally detected in the epithelial components in two cases, which are one ameloblastic fibroma and one ameloblastic fibro-odontoma [13]. While this positivity in the epithelial and mesenchymal components of these two samples may have occurred due to cross-contamination and detected using a high-sensitivity molecular technique, it may also represent the true mixed nature of such lesions.

\section{Odontogenic Sarcomas}

The molecular pathogenesis of odontogenic sarcomas, namely ameloblastic fibrosarcoma, has been explored recently [13, 75]. The BRAF p.V600E mutation has been detected in $71 \%$ $(5 / 7)$ [75] and $67 \%(2 / 3)$ [13] of cases. Consistent with the 
histopathologic features of the ameloblastic fibrosarcoma, in which malignancy is restricted to the mesenchymal component, the BRAF p.V600E mutation was restricted to the sarcomatous areas of the two mutant ameloblastic fibrosarcoma cases tested in our cohort [13]. The mutation was further detected in the benign mesenchymal component of the ameloblastic fibroma-like area in one of the ameloblastic fibrossarcoma positive cases [13]. This finding reinforces the malignant transformation from a previous ameloblastic fibroma in some cases [13]. In addition to BRAF mutations, NRAS p.Q61K has been detected in a BRAF wild-type ameloblastic fibrosarcoma sample [75].

Although the rarity of this tumor precludes extensive knowledge about its molecular pathology, the current results could support the role of the MAPK/ERK pathway in its pathogenesis and pave the way for further investigation on targeted therapy.

\section{Odontogenic Carcinomas}

Ameloblastic carcinomas, the malignant counterpart of ameloblastomas, also harbor BRAF p.V600E mutations, but with reported frequencies varying from 25 to $40 \%$ [10, 53, 63]. Such frequencies are lower than those reported for the same mutations for ameloblastomas (64\% in conventional, $81 \%$ in unicystic, and $63 \%$ in peripheral), as shown in Table 1 . It is worth noting that the lower frequency of $B R A F$ p.V600E mutation in ameloblastic carcinomas compared with ameloblastomas is similar to what is observed in other benign tumors and their malignant counterparts [6]. For instance, BRAF p.V600E mutation is detected in approximately $80 \%$ of benign melanocytic nevi, $60 \%$ of dysplastic nevi, and only in $40-45 \%$ of melanomas, suggesting that the functional effects of the mutation are context-dependent [6]. Additionally, oncogene-induced senescence may limit the proliferation status of nevi in the tumorigenic process [79]. However, BRAF p.V600E mutation has been recently reported in $5 / 5$ ameloblastic carcinoma cases submitted to molecular screening [76]. These results could encourage targeted therapy as a new direction in the future.

Mutations in other genes which are not related to MAPK/ERK, such as TP53, CTNNB1, and APC, have also been reported in ameloblastic carcinomas [72, 78, 80]. The detection of TP53 mutation in the malignant area of a tumor arising from preexisting ameloblastoma [80] and observation of TP53 and CTNNB1 in a BRAF p.V600E wild-type ameloblastic carcinoma [72] suggest that mutations in these genes might play a role in the malignant transformation process of ameloblastomas.

Odontogenic carcinoma with dentinoid, which is an odontogenic carcinoma that has not yet been fully recognized as a unique entity, has also been shown to harbor pathogenic mutation in CTNNB1 and APC [81]. Although these genes are not part of the MAPK/ERK pathway, they belong to WNT/ $\beta$ catenin, which is well known to cross-talk with MAPK/ERK. Inactivating $A P C$ mutation and CTNNB1 activating mutation leads to strong and aberrant cellular $\beta$-catenin accumulation $[37,38,78,81]$, and such phenomena have been observed in some odontogenic epithelial tumors, including ameloblastoma and odontogenic carcinoma $[78,81]$.
Clear cell odontogenic carcinoma has also been shown to harbor BRAF p.V600E [10]. However, since a single sample was screened, future studies including a larger cohort of samples may clarify if such mutations are frequent in this tumor.

\section{Adenomatoid Odontogenic Tumor}

An adenomatoid odontogenic tumor is a benign epithelial odontogenic tumor predominantly affecting the maxilla. It is often encapsulated and has an indolent clinical behavior $[1,82]$. Adenomatoid odontogenic tumors most often occur sporadically. However, multiple adenomatoid odontogenic tumors can occur in patients with Schimmelpenning syndrome (OMIM\#163200) [83, 84]. This syndrome is caused by postzygotic mutations in RAS genes [85]. Based on that knowledge, we screened an adenomatoid odontogenic tumor sample from a Schimmelpenning syndrome patient as well as two sporadic cases for mutations in a NGS panel comprising 50 tumor suppressor genes and oncogenes, including RAS family genes, by using Ion AmpliSeq ${ }^{\mathrm{TM}}$ Cancer Hotspot Panel v2 (Life Technologies, Carlsbad, USA). The KRAS c.35G > T mutation leading to p.G12V was detected in all three samples, and it was further interrogated in additional samples. The mutation was detected in $78 \%$ (7/9) of tumors evaluated [11].

Following the aforementioned study, we assessed KRAS codon 12 mutations by allele-specific qPCR and screened codons 12, 13 , and 61 by Sanger sequencing in a larger cohort of samples. We detected recurrent KRAS p.G12V $(n=15 / 38)$ or p.G12R mutations $(n=12 / 38)$ in 27 out of 38 adenomatoid odontogenic tumors $(71 \%)$ regardless of the age of the patient, tumor size, tumor location, follicular or extrafollicular variants, and fibrous capsule thickness [12]. Confirming our findings, the study of Bologna-Molina et al. also reported KRAS codon 12 mutations in $78 \%(7 / 9)$ of cases, with either p.G12V $(n=2 / 9)$ or p.G12R ( $n$ $=4 / 9)$ mutations reported in $6 / 9$ samples and p.G12D in a single case (1/9) [74].

Further than investigating point mutations, we have also investigated the copy-neutral loss of heterozygosity $(\mathrm{cnLOH})$, as well as copy number alterations (CNAs) in a sporadic adenomatoid odontogenic tumor and a sample from a Schimmelpenning syndrome patient [11]. The sporadic tumor showed two rare CNAs, being one at $6 \mathrm{p} 15$ and the other at $7 \mathrm{p} 15.3$, covering the IGF2BP3 gene, while the tumor from the syndromic patient only harbored common gains and losses [11]. The deletion only encompasses an intronic portion of the gene, and the significance of these findings in the context of tumorigenesis is unclear.

While the BRAF p.V600E mutation emerged as a molecular signature for ameloblastomas and has also been shown to be frequent in other tumors with ameloblastic differentiation, KRAS codon 12 mutations seem to be a marker of adenomatoid odontogenic tumors [11, 12, 74]. Even tumors wild-type for KRAS showed MAPK/ERK pathway activation, demonstrated by the immunoexpression of the surrogate marker ERK1/2 phosphorylated form [12]. Therefore, whole-exome sequencing of the KRAS wild-type cases may provide information on the genetic signatures of the remaining $30 \%$ of cases for which KRAS mutations have not been detected. 


\section{MOLECULAR PATHOLOGY AND TARGETED THERAPY}

Although the molecular pathogenesis of ameloblastomas has not been entirely elucidated, the high frequency of BRAF p.V600E mutation strongly supports such mutation as a therapeutic target in a large proportion of these tumors. The BRAF-mutant human cancers, e.g., melanomas, show a good response to BRAF-targeted inhibition [86]. In line with that, in vitro studies and case reports have focused on BRAF-targeted therapy in ameloblastomas [51, 52, 87-92]. Notably, ameloblastoma cell lines harboring BRAF p.V600E mutation seem to be sensitive to vemurafenib, i.e., a BRAF small molecule inhibitor, which inhibited cell proliferation and MAPK/ERK activation [51, 52]. Reduction in the tumor mass has also been reported by using dual therapy with BRAF/MEK inhibitors, i.e., dabrafenib and trametinib, in metastatic ameloblastomas [87, 91] and monotherapy, i.e., dabrafenib or vemurafenib, in recurrent [8890] and metastatic [92] ameloblastomas.

Despite the absence of recurrent MAPK/ERK pathogenic mutations in odontogenic myxomas [93], a pilot study demonstrated positive immunoexpression of ERK1/2 phosphorylated form suggesting MAPK/ERK pathway activation in odontogenic myxoma [94]. Moreover, in this study, MEK was investigated as a possible therapeutic target using the administration of U0126 (a MEK inhibitor), showing promising results both in in vitro and in vivo tests, which expresses a possible potential use of MEK inhibitors in aggressive cases of odontogenic myxomas [94]. However, considering the limitation of this pilot study, including limited sample numbers, such insights need further confirmation by additional reports.

It is worth noting that the successful results of the above-mentioned studies suggested that molecular targeted therapy is potentially a neoadjuvant treatment and could diminish morbidities related to radical surgery in aggressive and advanced cases of odontogenic tumors carrying MAPK/ERK activating mutations [95]. Nonetheless, the use of MAPKtargeted therapy is still debatable since conservative approaches have demonstrated a high recurrence rate and the use of BRAF/MEK inhibitors might lead to the development of drug resistance and/or serious adverse effects $[96,97]$. Further clinical trials addressing the use of BRAF, BRAF/MEK, and MEK inhibitors for odontogenic tumor treatment are necessary to clarify their effectiveness, the advantages and disadvantages, actual efficacy, and best treatment regimen for the patients. Therefore, molecular targeted therapy should not be applied in all MAPK-related odontogenic tumor cases but should be reserved for the most aggressive ones as the first step of treatment to reduce morbidity.

\section{CONCLUDING REMARKS}

There is still very limited information on the molecular pathogenesis of odontogenic tumors, with most of the studies focusing either on single genetic mutations or in small gene panels. The advances in molecular techniques for mutation screening have allowed a better understanding of the molecular basis of odontogenic tumors during the last decade. Although their pathogenesis has not been entirely elucidated, the detection of pathogenic mutations in MAPK/ERK pathway genes in some odontogenic tumors strongly suggested that this pathway constitutive activation contributed to tumorigenesis. In a physiological context, the MAPK/ERK signaling pathway regulated important cellular biological functions and plays a role in odontogenesis. Therefore, disturbances in this signaling pathway can lead to odontogenic tumor development.

Among the MAPK/ERK mutations reported in odontogenic tumors, the most prominent is BRAF p.V600E. This mutation is present in a high percentage of ameloblastomas, including conventional (64\%), unicystic (81\%), and peripheral (63\%) variants, being considered a molecular signature for this locally aggressive odontogenic tumor. In addition, $B R A F$ p.V600E has also been reported in benign mixed odontogenic tumors, namely, ameloblastic fibroma, ameloblastic fibrodentinoma, and ameloblastic fibro-odontoma, and in malignant odontogenic tumors, namely, ameloblastic carcinoma, ameloblastic fibrosarcoma, and clear cell odontogenic carcinoma. Importantly, KRAS p.G12V or p.G12R mutations have been reported in $\sim 70 \%$ of adenomatoid odontogenic tumors, an encapsulated and indolent epithelial odontogenic tumor. Therefore, the constitutive activation of the same MAPK/ERK pathway in odontogenic tumors with such distinct biological behaviors emphasizes that the functional effects of the pathogenic mutations that disrupt the pathway are context-dependent.

The identification and clarification of the role mutations in MAPK/ERK components play in odontogenic tumors is of core importance for translational application in this heterogeneous and complex group of lesions. Targeted therapy may be a useful tool in the treatment of aggressive and advanced odontogenic tumors. Although targeted therapy has mainly focused on ameloblastomas, further in vitro and in vivo studies, and clinical trials may help to establish standardized therapeutic regimens both for ameloblastomas and other BRAF mutation-positive odontogenic tumors. MAPK/ERK cross talks with other signaling pathways must also be considered in studies focusing on targeted therapy.

Besides being important for therapeutics, the elucidation of $B R A F$ and RAS mutations in odontogenic tumors might be helpful to diagnosis and classification. For instance, molecular screening can help to solve cases with challenging diagnoses. Moreover, the discovery of BRAF p.V600E mutation in ameloblastic fibromas, ameloblastic fibrodentinomas, and ameloblastic fibro-odontomas and the absence of such mutation in odontomas suggested that at least a subset of these tumors should not be classified as the early stages of odontoma.

Currently, these MAPK signaling pathway mutations are known to occur in specific odontogenic tumors, but the question is whether they are important in the initiation of these tumors. Furthermore, whether these mutations impact tumor evolution remains a question. The activation 
of the MAPK/ERK pathway is linked to cell senescence in other tumorigenic processes. This leads to the question if the same hold true for odontogenic tumors. Moreover, why ameloblastomas show a higher frequency of $B R A F$ mutation than their malignant counterpart, ameloblastic carcinomas, remains a question. Although several odontogenic tumors have recently been included in the spectrum of MAPK/ERK-driven tumors, further studies are needed to clarify the role such mutations play in the pathogenesis of odontogenic tumors.

\section{AUTHOR CONTRIBUTIONS}

CCG provided leadership for the project. LMG, BPC, and CCG performed data/evidence collection and analysis, wrote the

\section{REFERENCES}

1. El-Naggar AK, Chan John KC, Grandis JR, Takata T, Slootweg PJ. World Health Organization Classification of Head and Neck Tumours. 4th ed. Lyon, France: World Health Organization. (2017) p. 347.

2. Balic A. Thesleff, I. Tissue interactions regulating tooth development and renewal. Curr Top Dev Biol. (2015) 115:15786. doi: 10.1016/bs.ctdb.2015.07.006

3. Diniz MG, Gomes CC, de Sousa SF, Xavier GM, Gomez RS. Oncogenic signalling pathways in benign odontogenic cysts and tumours. Oral Oncol. (2017) 72:165-73. doi: 10.1016/j.oraloncology.2017.07.021

4. Lavoie H, Gagnon J, Therrien M. ERK signalling: a master regulator of cell behaviour, life and fate. Nat Ver Mol Cell Biol. (2020) 21:60732. doi: 10.1038/s41580-020-0255-7

5. Marino-Enriquez A, Fletcher CDM. Shouldn't we care about the biology of benign tumours? Nat Rev Cancer. (2014) 14:701-2. doi: 10.1038/nrc3845

6. Kato S, Lippman SM, Flaherty KT, Kurzrock R. The conundrum of genetic "Drivers" in benign conditions. J Natl Cancer Inst. (2016) 108:djw036. doi: 10.1093/jnci/djw036

7. Myers MB, Banda M, McKim KL, Wang Y, Powell MJ, Parsons BL. Breast Cancer heterogeneity examined by high-sensitivity quantification of PIK3CA, KRAS, HRAS, and BRAF mutations in normal breast and ductal carcinomas. Neoplasia. (2016) 18:253-63. doi: 10.1016/j.neo.2016. 03.002

8. Lac V, Nazeran TM, Tessier-Cloutier B, Aguirre-Hernandez R, Albert A, Lum A, et al. Oncogenic mutations in histologically normal endometrium: the new normal? J Pathol. (2019) 249:173-81. doi: 10.1002/path.5314

9. Kurppa KJ, Catón J, Morgan PR, Ristimäki A, Ruhin B, Kellokoski J, et al. High frequency of BRAF V600E mutations in ameloblastoma. J Pathol. (2014) 232:492-8. doi: 10.1002/path.4317

10. Diniz MG, Gomes CC, Guimarães BVA, de Castro WH, Lacerda JCT, Cardoso SV, et al. Assessment of BRAFV600E and SMOF412E mutations in epithelial odontogenic tumours. Tumour Biol. (2015) 36:564953. doi: 10.1007/s13277-015-3238-0

11. Gomes CC, de Sousa SF, de Menezes GHM, Duarte AP, Pereira TSF, Moreira RG, et al. Recurrent KRAS G12V pathogenic mutation in adenomatoid odontogenic tumours. Oral Oncol. (2016) 56:e3-5. doi: 10.1016/j.oraloncology.2016.03.001

12. Coura BP, Bernardes VF, de Sousa SF, França JA, Pereira NB, Pontes HAR, et al. KRAS mutations drive adenomatoid odontogenic tumor and are independent of clinicopathological features. Mod Pathol. (2019) 32:799806. doi: 10.1038/s41379-018-0194-4

13. Coura BP, Bernardes VF, de Sousa SF, Diniz MG, Moreira RG, de Andrade $\mathrm{BAB}$, et al. Targeted next-generation sequencing and allele-specific quantitative PCR of laser capture microdissected samples uncover molecular differences in mixed odontogenic tumors. J Mol Diagn. (2020) 22:13939. doi: 10.1016/j.jmoldx.2020.08.005 initial draft, and generated the text. LPG elaborated the figures. CCG and RSG critically revised the manuscript. All authors contributed to the final manuscript and had final approval of the submitted version.

\section{FUNDING}

This study received funding from The Coordination for the Improvement of Higher-Level Education Personnel (CAPES)/Brazil [finance code 001], Fundação de Amparo à Pesquisa do Estado de Minas Gerais (FAPEMIG)/Brazil and The National Council of Scientific and Technological Development $(\mathrm{CNPq}) / \mathrm{Brazil}$. LMG and $\mathrm{BPC}$ receive CAPES and $\mathrm{CNPq}$ scholarships, respectively. RSG and CCG are research fellows at CNPq.

14. Cargnello M, Roux PP. Activation and function of the MAPKs and their substrates, the MAPK-activated protein kinases. Microbiol Mol Biol Rev. (2011) 75:50-83. doi: 10.1128/MMBR.00031-10

15. Krishna $M$, Narang $H$. The complexity of mitogen-activated protein kinases (MAPKs) made simple. Cell Mol Life Sci. (2008) 65:352544. doi: 10.1007/s00018-008-8170-7

16. Crews CM, Alessandrini A, Erikson RL. The primary structure of MEK, a protein kinase that phosphorylates the ERK gene product. Science. (1992) 258:478-80. doi: 10.1126/science.1411546

17. Widmann C, Gibson S, Jarpe MB, Johnson GL. Mitogen-activated protein kinase: conservation of a three-kinase module from yeast to human. Physiol Rev. (1999) 79:143-80. doi: 10.1152/physrev.1999.79.1.143

18. Wellbrock C, Karasarides M, Marais R. The RAF proteins take centre stage. Nat Rev Mol Cell Biol. (2004) 5:875-85. doi: 10.1038/nrm1498

19. Yoon S, Seger R. The extracellular signal-regulated kinase: multiple substrates regulate diverse cellular functions. Growth Factors. (2006) 24:2144. doi: 10.1080/02699050500284218

20. Hoshino R, Chatani Y, Yamori T, Tsuruo T, Oka H, Yoshida O, et al. Constitutive activation of the $41-/ 43-\mathrm{kDa}$ mitogen-activated protein kinase signaling pathway in human tumors. Oncogene. (1999) 18:81322. doi: 10.1038/sj.onc. 1202367

21. Dhillon AS, Hagan S, Rath O, Kolch W. MAP kinase signalling pathways in cancer. Oncogene. (2007) 26:3279-90. doi: 10.1038/sj.onc.1210421

22. Ikawa S, Fukui M, Ueyama Y, Tamaoki N, Yamamoto T, Toyoshima K. B-raf, a new member of the raf family, is activated by DNA rearrangement. $\mathrm{Mol}$ Cell Biol. (1988) 8:2651-4. doi: 10.1128/mcb.8.6.2651-2654.1988

23. Michaloglou C, Vredeveld LCW, Mooi WJ, Peeper DS. BRAF(E600) in benign and malignant human tumours. Oncogene. (2008) 27:87795. doi: 10.1038/sj.onc. 1210704

24. Pratilas CA, Xing F, Solit DB. Targeting oncogenic BRAF in human cancer. Curr Top Microbiol Immunol. (2012) 355:83-98. doi: 10.1007/82_2011_162

25. Mercer K, Giblett S, Green S, Lloyd D, Dias SDR, Plumb M, et al. Expression of endogenous oncogenic V600EB-raf induces proliferation and developmental defects in mice and transformation of primary fibroblastos. Carcer Res. (2005) 65:11493-500. doi: 10.1158/0008-5472.CAN-05-2211

26. Davies H, Bignell GR, Cox C, Stephens P, Edkins S, Clegg S, et al. Mutations of the BRAF gene in human cancer. Nature. (2002) 417:94954. doi: 10.1038/nature00766

27. Dankner M, Rose AAN, Rajkumar S, Siegel PM, Watson IR. Classifying BRAF alterations in cancer: new rational therapeutic strategies for actionable mutations. Oncogene. (2018) 37:3183-99. doi: 10.1038/s41388-018-0171-x

28. Uguen A, Talagas M, Costa S, Samaison L, Paule L, Alavi Z, et al. NRAS (Q61R), BRAF (V600E) immunohistochemistry: a concomitante tool for mutation screening in melanomas. Diagn pathol. (2015) 10:121. doi: 10.1186/s13000-015-0359-0

29. Thiel A, Moza M, Kytöla S, Orpana A, Jahkola T, Hernberg $\mathrm{M}$, et al. Prospective immunohistochemical analysis of BRAF 
V600E mutation in melanoma. Hum Pathol. (2015) 46:16975. doi: 10.1016/j.humpath.2014.08.018

30. Capper D, Preusser M, Habel A, Sahm F, Ackermann U, Schindler G, et al. Assessment of BRAF V600E mutation status by immunohistochemistry with a mutation-specific monoclonal antibody. Acta Neuropathol. (2011) 122:119. doi: 10.1007/s00401-011-0841-z

31. Estrella JS, Tetzlaff MT, Bassett Jr RL, Patel KP, Williams MD, Curry JL, et al. Assessment of BRAF V600E status in colorectal carcinoma: tissue-specific discordances between immunohistochemistry and sequencing. Mol Cancer Ther. (2015) 14:2887-95. doi: 10.1158/1535-7163.MCT-15-0615

32. Hang JF Li AF, Chang SC, Liang WY. Immunohistochemical detection of the BRAF V600E mutant protein in colorectal cancers in Taiwan is highly concordant with the molecular test. Histopathology. (2016) 69:5462. doi: $10.1111 /$ his. 12903

33. Sasaki H, Shimizu S, Tani Y, Shitara M, Okuda K, Hikosaka Y, et al. Usefulness of immunohistochemistry for the detection of the BRAF V600E mutation in Japanese lung adenocarcinoma. Lung Cancer. (2013) 82:3154. doi: 10.1016/j.lungcan.2013.06.014

34. Haigis KM. KRAS Alleles: the devil is in the detail trends. Cancer. (2017) 3:686-97. doi: 10.1016/j.trecan.2017.08.006

35. Coura BP, Resende TAC, Menezes VCB, Bernardes VF, Sousa SF, Diniz MG, et al. Assessing pathogenic mutations in dental follicles as an attempt to identify early events in odontogenic tumours tumourigenesis. Arch Oral Biol. (2020) 113:104523. doi: 10.1016/j.archoralbio.2019.104523

36. Mendoza MC, Er EE, Blenis J. The Ras-ERK and PI3K-mTOR pathways: Cross-talk and compensation. Trends Biochem Sci. (2011) 36:320-8. doi: 10.1016/j.tibs.2011.03.006

37. Zhang Y, Pizzute T, Pei M. A review of crosstalk between MAPK and Wnt signals and its impact on cartilage regeneration. Cell Tissue Res. (2014) 358:633-49. doi: 10.1007/s00441-014-2010-x

38. Jeong W-J, Ro EJ, Choi K-Y. Interaction between Wnt/B-catenin and RASERK pathways and an anti-cancer strategy via degradations of $\beta$-catenin and RAS by targeting the Wnt/ $\beta$-catenin pathway. NPJ Precis Oncol. (2018) 2:5. doi: 10.1038/s41698-018-0049-y

39. Marangoni P, Charles C, Tafforeau P, Laugel-Haushalter V, Joo A, BlochZupan A, et al. Phenotypic and evolutionary implications of modulating the ERK-MAPK cascade using the dentition as a model. Sci Rep. (2015) 5:11658. doi: $10.1038 /$ srep 11658

40. Laugel-Haushalter V, Paschaki M, Marangoni P, Pilgram C, Langer A, Kuntz T, et al. RSK2 is a modulator of craniofacial development. PLoS ONE. (2014) 9:e84343. doi: 10.1371/journal.pone.0084343 eCollection 2014

41. Klein OD, Minowada G, Peterkova R, Kangas A, Yu BD, Lesot H, et al. Sprouty genes control diastema tooth development via bidirectional antagonism of epithelial-mesenchymal FGF signaling. Dev Cell. (2006) 11:181-90. doi: 10.1016/j.devcel.2006.05.014

42. Chen X, Mitsutake N, LaPerle K, Akeno N, Zanzonico P, Longo VA, et al. Endogenous expression of $\operatorname{Hras}(\mathrm{G} 12 \mathrm{~V})$ induces developmental defects and neoplasms with copy number imbalances of the oncogene. Proc Natl Acad Sci U S A. (2009) 106:7979-84. doi: 10.1073/pnas.0900343106

43. Goodwin AF, Tidyman WE, Jheon AH, Sharir A, Zheng X, Charles $\mathrm{C}$, et al. Abnormal Ras signaling in Costello syndrome (CS) negatively regulates enamel formation. Hum Mol Genet. (2014) 23:682-92. doi: $10.1093 / \mathrm{hmg} / \mathrm{ddt} 455$

44. Gibson CW, Lally E, Herold RC, Decker S, Brinster RL, Sandgren EP. Odontogenic tumors in mice carrying albumin-myc and albumin-rats transgenes. Calcif Tissue Int. (1992) 51:162-7. doi: 10.1007/BF00298506

45. Cardiff RD, Leder A, Kuo A, Pattengale PK, Leder P. Multiple tumor types appear in a transgenic mouse with the ras oncogene. Am J Pathol. (1993) 142:1199-207.

46. Wright JT, Hansen L, Mahler J, Szczesniak C. Spalding JW. Odontogenic tumours in the v-Ha-ras (TGAC) transgenic mouse. Arch Oral Biol. (1995) 40:631-8. doi: 10.1016/0003-9969(95)00017-j

47. Kumamoto H, Takahashi N, Ooya K. K-Ras gene status and expression of Ras/mitogen-activated protein kinase (MAPK) signaling molecules in ameloblastomas. J Oral Pathol Med. (2004) 33:360-7. doi: 10.1111/j.1600-0714.2004.00141.x

48. Kettunen P, Karavanova I, Thesleff I. Responsiveness of developing dental tissues to fibroblast growth factors: expression of splicing alternatives of FGFR1,-2,-3, and of FGFR4; and stimulation of cell proliferation by FGF-2,-4,-8, and-9. Dev Genet. (1998) 22:37485. doi: 10.1002/(SICI) 1520-6408(1998)22:4<374::AID-DVG7>3.0.CO;2-3

49. Kettunen $P$, Laurikkala J, Itäranta $P$, Vainio $S$, Itoh $N$, Thesleff I. Associations of FGF-3 and FGF-10 with signaling networks regulating tooth morphogenesis. Dev Dyn. (2000) 219:32232. doi: 10.1002/1097-0177(2000)9999:9999<::AID-DVDY1062>3.0.CO;2-J

50. Davideau JL, Sahlberg C, Blin C, Papagerakis P, Thesleff I, Berdal A. Differential expression of the full-length and secreted truncated forms of EGF receptor during formation of dental tissues. Int J Dev Biol. (1995) 39:605-15.

51. Sweeney RT, McClary AC, Myers BR, Biscocho J, Neahring L, Kwei KA, et al. Identification of recurrent $\mathrm{SMO}$ and BRAF mutations in ameloblastomas. Nat Genet. (2014) 46:722-5. doi: 10.1038/ng.2986

52. Brown NA, Rolland D, McHugh JB, Weigelin HC, Zhao L, Lim MS, et al. Activating FGFR2-RAS-BRAF mutations in ameloblastoma. Clin Cancer Res. (2014) 20:5517-26. doi: 10.1158/1078-0432.CCR-14-1069

53. Brunner P, Bihl M, Jundt G, Baumhoer D. Hoeller S. BRAF pV600E mutations are not unique to ameloblastoma and are shared by other odontogenic tumors with ameloblastic morphology. Oral Oncol. (2015) 51:e77-78. doi: 10.1016/j.oraloncology.2015.07.010

54. Yukimori A, Oikawa Y, Morita K, Nguyen CTK, Harada H, Yamaguchi S, et al. Genetic basis of calcifying cystic odontogenic tumors. PLoS ONE. (2017) 28:e0180224. doi: 10.1371/journal.pone.0180224

55. Gültekin SE, Aziz R, Heydt C, Sengüven B, Zöller J, Safi AF, et al. The landscape of genetic alterations in ameloblastomas relates to clinical features. Virchows Arch. (2018) 472:807-14. doi: 10.1007/s00428-018-2305-5

56. Soltani M, Tabatabaiefar MA, Mohsenifar Z, Pourreza MP, Moridnia A, Shariati $L$, et al. Genetic study of the BRAF gene reveals new variants and high frequency of the V600E mutation among Iranian ameloblastoma patients. $J$ Oral Pathol Med. (2018) 47:86-90. doi: 10.1111/jop.12610

57. Guan P, Wong SF, Lim JQ, Ng CCY, Soong PL, Sim CQX, et al. Mutational signatures in mandibular ameloblastoma correlate with smoking. J Dent Res. (2019) 98:652-8. doi: 10.1177/002203451983724

58. Heikinheimo K, Huhtala J-M, Thiel A, Kurppa KJ, Heikinheimo H, Kovac M, et al. The mutational profile of unicystic ameloblastoma. J Dent Res. (2019) 98:54-60. doi: 10.1177/0022034518798810

59. Derakhshan S, Aminishakib P, Karimi A, Saffar H, Abdollahi A, Mohammadpour H, et al. High frequency of BRAF V600E mutation in Iranian population ameloblastomas. Med Oral Patol Oral Cir Bucal. (2020) 25:e502-7. doi: 10.4317/medoral.23519

60. Seki-Soda M, Sano T, Ito K, Yokoo S, Oyama T. An immunohistochemical and genetic study of BRAFV600E mutation in Japanese patients with ameloblastoma. Pathol Int. (2020) 70:224-30. doi: 10.1111/pin. 12899

61. Zhang R, Yang Q, Qu J, Hong Y, Liu P. Li T. The BRAF pV600E mutation is a common event in ameloblastomas but is absent in odontogenic keratocysts. Oral Surg Oral Med Oral Pathol Oral Radiol. (2020) 129:22935. doi: 10.1016/j.oooo.2019.04.001

62. Bonacina R, Indini A, Massazza G, Rulli E, Gianatti A, Mandalà M, et al. Correlation of BRAF mutational status with clinical characteristics and survival outcomes of patients with ameloblastoma: the experience of 11 Italian centres. J Clin Pathol. (2021) 0:1-5. doi: 10.1136/jclinpath-2021-207527

63. Oh K-Y, Cho S-D, Yoon H-J, Lee J-I, Hong S-D. Discrepancy between immunohistochemistry and sequencing for BRAF V600E in odontogenic tumours: Comparative analysis of two VE1 antibodies. J Oral Pathol Med. (2021) 50:85-91. doi: 10.1111/jop.13108

64. Sant'Ana MSP, Costa SFS, da Silva MP, Martins-Chaves RR, Pereira TSF, de Oliveira EM, et al. BRAF p.V600E status in epithelial areas of ameloblastoma with different histological aspects: Implications to the clinical practice. J Oral Pathol Med. (2021) 50:478-84. doi: 10.1111/jop.13155

65. Shi Y, Li M, Yu Y, Zhou Y, Wang S. Whole exome sequencing and system biology analysis support the "twohit" mechanism in the onset of Ameloblastoma. Med Oral Patol Oral Cir Bucal. (2021) 26:e510-7. doi: 10.4317/medoral. 24385

66. Fregnani ER, Perez DEC, de Almeida OP, Fonseca FP, Soares FA, CastroJunior G, et al. BRAF-V600E expression correlates with ameloblastoma aggressiveness. Histopathology. (2017) 70:473-84. doi: 10.1111/his.13095 
67. Shirsat PM, Bansal S, Prasad P, Desai RS. Low frequency of BRAF V600E immunoexpression in mandibular ameloblastomas: An institutional study. $J$ Oral Maxillofac Pathol. (2018) 22:353-9. doi: 10.4103/jomfp.JOMFP_174_17

68. do Canto AM, Marcelino BMRS, Schussel JL, Wastner BF, Sassi LM, Corrêa L, et al. Immunohistochemical analysis of BRAF V600E mutation in ameloblastomas. Clin Oral Investig. (2019) 23:779-84. doi: 10.1007/s00784-018-2494-y

69. Kelppe J, Thorén H, Ristimäki A, Haglund C, Sorsa T, Hagström J, et al. V600E expression in ameloblastomas-A 36-patient cohort from Helsinki University Hospital. Oral Dis. (2019) 25:1169-74. doi: 10.1111/odi.13072

70. Lapthanasupkul P, Laosuk T, Ruangvejvorachai P, Aittiwarapoj A, Kitkumthorn N. Frequency of BRAF V600E mutation in a group of Thai patients with ameloblastomas. Oral Surg Oral Med Oral Pathol Oral Radiol. (2020). doi: 10.1016/j.oooo.2020.06.002. [Epub ahead of print].

71. Owosho AA, Ladeji AM, Adebiyi KE, Olajide MA, Okoye ISI, Kehinde $\mathrm{T}$, et al. BRAF V600E mutation-specific immunohistochemical analysis in ameloblastomas: a 44-patient cohort study from a single institution. Eur Arch Otorhinolaryngol. (2020) 278:3065-71. doi: 10.1007/s00405-020-06491-w

72. Bartels S, Adisa A, Aladelusi T, Lemound J, Stucki-Koch A, Hussein $\mathrm{S}$, et al. Molecular defects in BRAF wild-type ameloblastomas and craniopharyngiomas-differences in mutation profiles in epithelial-derived oropharyngeal neoplasms. Virchows Arch. (2018) 472:1055-9. doi: 10.1007/s00428-018-2323-3

73. Pereira NB, Pereira KMA, Coura BP, Diniz MG, de Castro WH, Gomes CC, et al. BRAFV600E mutation in the diagnosis of unicystic ameloblastoma. $J$ Oral Pathol Med. (2016) 45:780-5. doi: 10.1111/jop.12443

74. Bologna-Molina R, Ogawa I, Mosqueda-Taylor A, Takata T, Sánchez-Romero C, Villarroel-Dorrego M, et al. Detection of MAPK/ERK pathway proteins and KRAS mutations in adenomatoid odontogenic tumors. Oral Dis. (2019) 25:481-7. doi: 10.1111/odi.12989

75. Agaimy A, Skalova A, Franchi A, Alshagroud R, Gill AJ, Stoehr R, et al. Ameloblastic fibrosarcoma: clinicopathological and molecular analysis of seven cases highlighting frequent BRAF and occasional NRAS mutations. Histopathology. (2020) 76:814-21. doi: 10.1111/his.14053

76. Niu Z, Li Y, Chen W, Zhao J, Zheng H, Deng Q, et al. Study on clinical and biological characteristics of ameloblastic carcinoma. Orphanet J Rare Dis. (2020) 15:316. doi: 10.1186/s13023-020-01603-5

77. Nakao Y, Mitsuyasu T, Kawano S, Nakamura N, Kanda S, Nakamura S. Fibroblast growth factors 7 and 10 are involved in ameloblastoma proliferation via the mitogen-activated protein kinase pathway. Int J Oncol. (2013) 43:1377-84. doi: 10.3892/ijo.2013.2081

78. Siriwardena BSMS, Kudo Y, Ogawa I, Tilakaratne WM, Takata T. Aberrant beta-catenin expression and adenomatous polyposis coli gene mutation in ameloblastoma and odontogenic carcinoma. Oral Oncol. (2009) 45:1038. doi: 10.1016/j.oraloncology.2008.03.008

79. Mooi WJ, Peeper DS. Oncogene-induced cell senescence-halting on the road to cancer. N Engl J Med. (2006) 355:1037-46. doi: 10.1056/NEJMra062285

80. Nobusawa A, Sano T, Yokoo S, Oyama T. Ameloblastic carcinoma developing in preexisting ameloblastoma with a mutation of the p53 gene: a case report. Oral Surg Oral Med Oral Pathol Oral Radiol. (2014) 118:e146150. doi: 10.1016/j.oooo.2014.03.021

81. Gondak RO, Mariano FV, Sousa SF, Siqueira EC, Díaz KP, Martins LAL, et al. CTNNB1 and APC mutations in odontogenic carcinoma with dentinoid. 2019. Oral Surg Oral Med Oral Pathol Oral Radiol. (2020) 129:e24956. doi: 10.1016/j.oooo.2019.08.017

82. Chrcanovic BR, Gomez RS. Adenomatoid odontogenic tumor: An updated analysis of the cases reported in the literature. J Oral Pathol Med. (2019) 48:10-6. doi: 10.1111/jop.12783

83. Ernst LM, Quinn PD, Alawi F. Novel oral findings in Schimmelpenning syndrome. Am J Med Genet A. (2007) 143A:881-3. doi: 10.1002/ajmg.a.31663

84. Martins-Chaves RR, Júnior AACP, Gomes CC, de Castro WH, Gomez RS. Multiple adenomatoid odontogenic tumors in a patient with Schimmelpenning syndrome. Oral Surg Oral Med Oral Pathol Oral Radiol. (2020) 129:e12-7. doi: 10.1016/j.oooo.2019.06.006

85. Groesser L, Herschberger E, Ruetten A, Ruivenkamp C, Lopriore E, Zutt $\mathrm{M}$, et al. Postzygotic HRAS and KRAS mutations cause nevus sebaceous and Schimmelpenning syndrome. Nat Genet. (2012) 44:7837. doi: $10.1038 / \mathrm{ng} .2316$

86. Vanella V, Festino L, Trojaniello C, Vitale MG, Sorrentino A, Paone $\mathrm{M}$, et al. The role of BRAF-targeted therapy for advanced melanoma in the immunotherapy era. Curr Oncol Rep. (2019) 21:76. doi: 10.1007/s11912-019-0827-x

87. Kaye FJ, Ivey AM, Drane WE, Mendenhall WM, Allan RW. Clinical and radiographic response with combined BRAF-targeted therapy in stage 4 ameloblastoma. J Natl Cancer Inst. (2014) 107:378. doi: 10.1093/jnci/dju378

88. Faden DL, Algazi A. Durable treatment of ameloblastoma with single agent BRAFi Re: Clinical and radiographic response with combined BRAFtargeted therapy in stage 4 ameloblastoma. J Natl Cancer Inst. (2016) 109:djw190. doi: 10.1093/jnci/djw190

89. Tan S, Pollack JR, Kaplan MJ, Colevas AD, West RB, BRAF. inhibitor treatment of primary BRAF-mutant ameloblastoma with pathologic assessment of response. Oral Surg Oral Med Oral Pathol Oral Radiol. (2016) 122:e5-7. doi: 10.1016/j.oooo.2015.12.016

90. Fernandes GS, Girardi DM, Bernardes JPG, Fonseca FP, Fregnani ER. Clinical benefit and radiological response with BRAF inhibitor in a patient with recurrent ameloblastoma harboring V600E mutation. BMC Cancer. (2018) 18:887. doi: 10.1186/s12885-018-4802-y

91. Brunet M, Khalifa E, Italiano A. Enabling precision medicine for rare head and neck tumors: the example of BRAF/MEK targeting in patients with metastatic ameloblastoma. Front Oncol. (2019) 9:1204. doi: 10.3389/fonc.2019.01204

92. Broudic-Guibert M. Blay J-Y,Vazquez L, Evrard A, Karanian M, Taïeb S, et al. Persistent response to vemurafenib in metastatic ameloblastoma with BRAF mutation: a case report. J Med Case Rep. (2019) 13:245. doi: 10.1186/s13256-019-2140-6

93. Santos JN, Neto ESS, França JA, Diniz MG, Moreira RG, de Castro WH, et al. Next-generation sequencing of oncogenes and tumor suppressor genes in odontogenic myxomas. J Oral Pahol Med. (2017) 46:10369. doi: $10.1111 /$ jop. 12598

94. Pereira NB, Bastos VC, de Souza JC, Diniz MG, Vitório JG, Kitten GT, et al. First insights for targeted therapies in odontogenic myxoma. Clin Oral Investig. (2020) 24:2451-8. doi: 10.1007/s00784-019-0 3107-4

95. Gomes CC, Diniz MG, Gomez RS. Progress towards personalized medicine for ameloblastoma. J Pathol. (2014) 232:488-91. doi: 10.1002/pat h.4331

96. You Z, Liu S-P, Du J, Wu Y-H, Zhang S-Z. Advancements in MAPK signaling pathways and MAPK-targeted therapies for ameloblastoma: A review. J Oral Pathol Med. (2019) 48:201-5. doi: 10.1111/jop. 12807

97. Shi HA, Ng CWB, Kwa CT, Sim QXC. Ameloblastoma: A succinct review of the classification, genetic understanding and novel molecular targeted therapies. Surgeon. (2020) 19:238-43. doi: 10.1016/j.surge.2020. 06.009

Conflict of Interest: The authors declare that the research was conducted in the absence of any commercial or financial relationships that could be construed as a potential conflict of interest.

Publisher's Note: All claims expressed in this article are solely those of the authors and do not necessarily represent those of their affiliated organizations, or those of the publisher, the editors and the reviewers. Any product that may be evaluated in this article, or claim that may be made by its manufacturer, is not guaranteed or endorsed by the publisher.

Copyright (c) 2021 Guimarães, Coura, Gomez and Gomes. This is an open-access article distributed under the terms of the Creative Commons Attribution License (CC $B Y)$. The use, distribution or reproduction in other forums is permitted, provided the original author(s) and the copyright owner(s) are credited and that the original publication in this journal is cited, in accordance with accepted academic practice. No use, distribution or reproduction is permitted which does not comply with these terms. 